\title{
Multiple stents in pancreatic strictures of chronic pancreatitis: is it worth the effort?
}

\section{(웅 $\circledast$}

\author{
Author \\ Guido Costamagna ${ }^{1,2}$ \\ Institutions \\ 1 Digestive Endoscopy Unit, Fondazione Policlinico \\ Universitario A. Gemelli IRCCS, Roma, Italy \\ 2 Università Cattolica S. Cuore, Roma, Italy, CERTT \\ (Centre for Endoscopic Research Therapeutics and \\ Training)
}

\author{
Bibliography \\ DOI https://doi.org/10.1055/a-1006-2708 | \\ Endoscopy International Open 2019; 07: E1699-E1700 \\ (c) Georg Thieme Verlag KG Stuttgart · New York \\ eISSN 2196-9736
}

Corresponding author

Guido Costamagna,

Fax: +390630156581

Guido.Costamagna@Unicatt.it
Persistent or recurrent pain dominates the clinical scenario in the natural history of chronic pancreatitis. The origin of pain in chronic pancreatitis may have numerous origins, however, it very often results as a consequence of pancreatic ductal hypertension secondary to obstruction of the main pancreatic duct, due to presence of stones, fibrotic strictures, or a combination of both.

The first attempt at endoscopic treatment of chronic pancreatitis was reported by Cremer in 1976 [1], when he performed a pancreatic sphincterotomy in a patient with "acute pancreatic cholangitis" due to impaction of a pancreatic stone into the major papilla causing biliary obstruction. Since then, most of the endoscopic therapeutic innovations in the field of chronic pancreatitis have taken place in Brussels, thanks to the innovative vision of Michel Cremer and his co-workers. Namely, the first stent to overcome a main pancreatic duct stricture was also placed in Brussels in 1985 [1].

In our experience, placement of pancreatic stents is required in approximately $50 \%$ of patients undergoing endoscopic treatment of chronic pancreatitis. Common practice entails use of one large-bore plastic stent (8.5-10 Fr) with a length tailored according to the location of the stricture. Stents are usually left in place for at least 1 year, and eventually replaced if the stricture has not resolved and is still symptomatic [2,3]. Approximately one-third of patients require protracted stenting. In this subset, more aggressive stenting might increase the chances of stricture disappearance, and therefore, improve the outcome.

Two alternatives are available to increment the radial dilating force: increasing the number of side-by-side plastic stents or placing a fully-covered, removable self-expanding metal stent. The latter option has produced conflicting results [4, 5], and is still under evaluation in prospective clinical trials (NCT02802020). Placement of multiple plastic stents for unrelenting ductal strictures of chronic pancreatitis was proposed by our group in 2001 [6], in analogy with the strategy that is currently widely accepted for postoperative biliary strictures [7].

In this issue of EIO, Papalavrentios et al. [8], once again from the Erasme Hospital in Brussels, report on a retrospective, comparative evaluation of results of plastic stenting in three groups of patients:1) single stent; 2) step-up with one and then two stents; and 3) exclusively two stents. Their conclusion is that two stents are not superior to a single stent or to a step-up approach.

We agree that the majority of patients undergoing stenting do not need more than one stent, and that a step-up approach should be adopted only in those strictures that do not respond after 1 year of single stent placement. However, the better results we obtained with the multiple stent strategy compared to the outcomes reported by Papalavrentios et al. may have some explanations. First, the average number of stents in our experience is three (range 2-4), which might explain a higher percentage of morphological success in stricture disappearance (around $90 \%$ ). Second, a much higher incidence of alcoholics in the Brussels cohort (67\% vs. $31 \%$ ) can also explain the lower response to double stenting: alcohol abuse increases the fibrotic component in chronic pancreatitis, and therefore, might reduce the success rate of mechanical dilation. Third, we always employed a gradual increase in number and caliber of plastic stents (from 8.5 to 10 , and, rarely, to $11.5 \mathrm{Fr}$ ) to achieve pro- 
gressive dilation of the stricture, following the same strategy as for postoperative biliary strictures.

\section{Conclusion}

In conclusion, while waiting for the ideal self-expanding metal stent for pancreatic strictures, a step-up approach with increasing number and caliber of plastic stents might allow an increase in the final success rate for endotherapy in the subset of patients with refractory pancreatic strictures after 1 year of single stent placement. The evidence is very low, but it will be very difficult to increase it, due to the small number of patients that can benefit from such a therapeutic strategy.

\section{Competing interests}

Cook Endoscopy: Grant/Research support. Olympus: Member on advisory committees or review panels. Boston Scientific: Member on advisory committees or review panels.

\section{References}

[1] Cremer M, Devière J, Delhaye M et al. Stenting in severe chronic pancreatitis: results of medium-term follow-up in seventy-six patients. Endoscopy 1991; 23: 171-176

[2] Eleftheriadis N, Dinu F, Delhaye M et al. Long-term outcome after pancreatic stenting in severe chronic pancreatitis. Endoscopy 2005; 37: $223-230$

[3] Dumonceau JM, Delhaye M, Tringali A et al. Endoscopic treatment of chronic pancreatitis: European Society of Gastrointestinal Endoscopy (ESGE) guideline - Updated August 2018. Endoscopy 2019; 51: 179193

[4] Korpela T, Udd M, Lindstrom O et al. Fully covered self-expanding metal stents for benign refractory pancreatic duct stricture in chronic pancreatitis. Scand J Gastroenterol 2019; 54: 365-370

[5] Tringali A, Vadalà di Prampero SF et al. Fully covered self-expandable metal stents to dilate persistent pancreatic strictures in chronic pancreatitis: long-term follow-up of a prospective study. Gastrointest Endosc 2018; 88: 939-946

[6] Costamagna G, Pandolfi M, Mutignani M et al. Long-term results of endoscopic management of postoperative bile duct strictures with increasing number of stents. Gastrointest Endosc 2001; 54: 162 - 168

[7] Dumonceau JM, Tringali A, Papanikolaou IS et al. Endoscopic biliary stenting: choice of stents and results: European Society of Gastrointestinal Endoscopy (ESGE) clinical guideline - Updated October 2017. Endoscopy 2018; 50: 910 - 930

[8] Papalavrentios L, Musala C, Gkolfakis P et al. Multiple stents are not superior to single stent insertionfor pain relief in patients with chronic pancreatitis: a retrospective comparative study. Endosc Int Open 2019; 07: E1595-E1604 